\title{
Transatlantica
}

Revue d'études américaines. American Studies Journal

1 | 2018

Slavery on Screen / American Women Writers Abroad: 1849-1976

\section{« Dorothea Tanning, le modernisme et l'avant- garde transatlantique »}

Conférence d'Alyce Mahon, Maîtresse de conférences en histoire de l'art moderne et contemporain à l'Université de Cambridge

« Giacometti Lab », Institut Giacometti, Paris, 7 juin 2019

\section{Yasna Bozhkova}

\section{(2) OpenEdition}

\section{Journals}

Édition électronique

URL : https://journals.openedition.org/transatlantica/11466

DOI : $10.4000 /$ transatlantica. 11466

ISSN : 1765-2766

Éditeur

Association française d'Etudes Américaines (AFEA)

Référence électronique

Yasna Bozhkova, « «Dorothea Tanning, le modernisme et l'avant-garde transatlantique » »,

Transatlantica [En ligne], 1 | 2018, mis en ligne le 30 septembre 2019, consulté le 10 février 2023. URL : http://journals.openedition.org/transatlantica/11466; DOI : https://doi.org/10.4000/transatlantica. 11466

Ce document a été généré automatiquement le 10 février 2023.

\section{(i) $\odot$

Creative Commons - Attribution - Pas d'Utilisation Commerciale - Pas de Modification 4.0 International - CC BY-NC-ND 4.0

https://creativecommons.org/licenses/by-nc-nd/4.0/ 


\section{« Dorothea Tanning, le modernisme et l'avant-garde transatlantique »}

Conférence d'Alyce Mahon, Maîtresse de conférences en histoire de l'art moderne et contemporain à l'Université de Cambridge

« Giacometti Lab », Institut Giacometti, Paris, 7 juin 2019

\section{Yasna Bozhkova}

1 Contrairement à ce que l'on pourrait penser, à l'Institut Giacometti ${ }^{1}$ sont souvent organisées des expositions ou des conférences qui touchent au domaine de l'art américain et sont susceptibles d'intéresser les anglicistes ${ }^{2}$. Le 7 juin 2019, Alyce Mahon, Maîtresse de conférences en histoire de l'art moderne et contemporain à l'Université de Cambridge, a donné une conférence en anglais, consacrée au parcours de la peintre surréaliste américaine Dorothea Tanning. Le propos était riche et passionnant. Alyce Mahon est également commissaire de l'exposition Dorothea Tanning, première rétrospective d'envergure consacrée à l'artiste, que le public a pu découvrir au Museo Reina Sofia de Madrid (3 octobre 2018-7 janvier 2019) puis à la Tate Modern de Londres (27 février-6 juin 2019).

2 L'intervention d'Alyce Mahon a porté sur le parcours transatlantique de Tanning, qui a passé une grande partie de sa vie en France, et sur sa façon d'évoluer dans les cercles avant-gardistes, comme en témoignent les dialogues artistiques qu'elle entretient avec d'autres artistes: Leonor Fini, Max Ernst, Marcel Duchamp entre autres. Elle s'est également interrogée sur les raisons pour lesquelles Tanning est souvent marginalisée, voire exclue des expositions portant sur l'art surréaliste. Alyce Mahon y voit deux explications. D'une part, ce serait parce que Tanning crée une forme de surréalisme très personnelle et singulière, qui met à l'épreuve le surréalisme orthodoxe ${ }^{3}$. Son œuvre continue d'ailleurs à évoluer bien au-delà de la fin du surréalisme " historique » en 1969, devenant progressivement plus abstraite. D'autre part, explique Mahon, ce serait aussi dû au fait que les femmes dans les mouvements avant-gardistes sont souvent marginalisées. En outre, Tanning elle-même avait une certaine réticence envers l'étiquette «femme artiste $»^{4}$ et envers le féminisme, ce qui complique les lectures 
féministes de son œuvre: "There is no such thing as a woman artist. You may be a woman and you may be an artist, but the one is a given and the other is you. »

Dans la première partie de sa conférence, Mahon a retracé le parcours biographique de Tanning, revenant notamment sur ses débuts artistiques, telles qu'elle les relate ellemême dans son autobiographie Birthday: née dans la petite ville de Galesburg, dans l'Illinois, où, selon Tanning elle-même, "nothing happens but the wallpaper », elle rêvait déjà à l'âge de sept ans d'être artiste et de vivre à Paris. Après un passage à l'Institut d'art de Chicago, elle s'installe à New York, où elle travaille comme dessinatrice publicitaire et vit un peu à la dérive jusqu'à la découverte, décisive pour elle, de l'exposition Fantastic Art, Dada, Surrealism, organisée par Alfred Barr au MoMA en 1936. Tanning décrit comme une épiphanie cette exposition qui l'initie aux œuvres des surréalistes comme Leonor Fini ou encore Max Ernst, et découvre dans le surréalisme un monde de possibilités infinies. Mahon a expliqué que la réputation médiatique de Leonor Fini, baptisée "a female of the Surrealist species" dans un article de presse de l'époque et célèbre pour son cosmopolitisme et sa remarquable beauté physique, a pu servir de modèle à Tanning. Afin d'illustrer à quel point cette exposition a laissé son empreinte sur l'imagination de l'artiste, Mahon a montré qu'une de ses œuvres beaucoup plus tardives, Pincushion to Serve as Fetish $(1965)^{5}$, s'inspire de la célèbre œuvre de Meret Oppenheim Object: Breakfast in Fur (1936), qui était présentée dans le cadre de l'exposition et qui fit grande impression sur le public. Selon Mahon, dans les deux œuvres, un objet domestique, une tasse à thé chez Oppenheim et une pelote à épingles chez Tanning, se transforme en fétiche, à travers l'utilisation de matériaux souples pour créer une sensation érotique et une envie de toucher l'objet insolite.

4 Alyce Mahon a insisté ensuite sur l'ironie de l'histoire qui sous-tend la première traversée transatlantique ${ }^{6}$ de Tanning: impressionnée par l'exposition au MoMA, Tanning prend le bateau en 1939 pour aller en France et rencontrer tous les surréalistes en personne, mais après l'éclatement de la Seconde guerre mondiale elle sera vite forcée de retourner aux États-Unis. Découverte par le galeriste Julien Levy en 1941, elle pourra quand même rencontrer, grâce à lui, les surréalistes qui se sont exilés à New York entre temps. En 1943, son tableau Birthday et sa nouvelle "Blind Date ${ }^{7}$ seront publiés dans la revue surréaliste VVV lancée par André Breton à New York: selon Mahon, l'inclusion de ces œuvres est un choix stratégique dans le repositionnement du surréalisme comme mouvement plus international. En 1944, Tanning aura sa première exposition en solo dans la galerie surréaliste de Levy. Comme l'a souligné Mahon, il était assez exceptionnel pour une artiste encore très peu connue d'avoir une exposition en solo dans la cette célèbre galerie et elle se trouva ainsi tout de suite sur la carte de l'art avant-gardiste.

5 Dans la deuxième partie de sa conférence, Alyce Mahon s'est attardée sur quelques-uns des tableaux les plus marquants de Tanning, en donnant des pistes d'interprétation. Selon elle, si l'on tend souvent à interpréter les œuvres des femmes dans les mouvements avant-gardistes de manière biographique, chez Tanning cette approche s'avère limitée, puisque l'artiste déclarait elle-même : « One of my reasons for painting was really to escape biography. » Elle a insisté sur l'importance du motif de la porte, et notamment des séries de portes qui s'ouvrent à l'infini ${ }^{8}$, déjà présent dans son plus célèbre autoportrait Birthday de 1942, qui célèbre sa découverte du surréalisme et son entrée dans ce monde de possibilités infinies9. Elle explique que pour Tanning, un 
tableau doit laisser la porte ouverte à l'imagination et créer des passages d'une réalité à l'autre, tissant une sorte de toile enchantée autour du spectateur.

6 Alyce Mahon a proposé une lecture très fine et nuancée de la dimension genrée de l'art de Tanning et de la façon dont sa vision se situe par rapport au surréalisme plus " masculin », y compris celui de Max Ernst. D'une part, elle a insisté sur l'importance de l'érotisme chez Tanning, qui donne vie aux phantasmes de la femme. Par exemple, elle a expliqué que dans le tableau The Guest Room (1950-52), Tanning crée des phantasmes qui s'approprient et détournent le fétiche surréaliste de la femme enfant, mais aussi critiquent le climat répressif aux États-Unis pendant les années 1950, et particulièrement l'atmosphère " aseptisée » créée par la censure de l'érotique. D'autre part, Mahon a souligné que Tanning voyait le travail de Max Ernst, notamment, comme un grand hommage au féminin. Elle a également précisé que des tableaux comme Eine Kleine Nachtmusik (1943) et The Guest Room, qui s'approprient la figure de la femme enfant, entretiennent un dialogue avec Ernst et ses représentations d'Alice, notamment dans The Stolen Mirror (1941). Dans sa lecture du tableau Self-Portrait de 1944, Mahon a avancé l'hypothèse que cette œuvre n'est pas, comme l'ont suggéré certains, une représentation de Tanning elle-même rendue insignifiante, voire écrasée, par sa relation avec Ernst et par l'immensité de l'art du peintre; ce tableau exprimerait plutôt, selon elle, une expérience du sublime dans le désert américain, inspirée par leur séjour à Sedona, dans l'Arizona, et relèverait d'une esthétique qui se situe dans la lignée des tableaux romantiques de Caspar David Friedrich Wanderer above the Sea of Fog et Woman before the Rising Sun. Elle a contesté l'idée selon laquelle les femmes artistes n'auraient pas besoin d'une muse, contrairement aux hommes, en prenant pour exemple le tableau Max in a Blue Boat (1947), où la figure d'Ernst apparaît comme celle d'une muse. Pour Tanning, sa relation avec Ernst se situait dans le cadre d'une égalité acquise entre les sexes : elle voyait Ernst comme un pair, le décrivant même comme un «fellow laboratory worker».

7 Pour finir, Alyce Mahon s'est attardée sur l'installation Hôtel du Pavot, Chambre 202 (1970-73), l'œuvre la plus monumentale de Tanning, mais aussi la plus surréaliste, selon l'artiste elle-même. Elle a précisé que cette chambre secrète, cet espace surréel qui semble être " partout et nulle part » à la fois, doit en partie son pouvoir de suggestion aux allusions qu'on peut y déceler : interprétée par certains comme une réponse à l'essai de Virginia Woolf A Room of One's Own, cette œuvre semble s'inspirer de sources hétéroclites, mêlant les influences artistiques, comme le poème en prose de Baudelaire «La Chambre double", la grande installation posthume de Marcel Duchamp Étant donnés, les poupées de Hans Bellmer, et les sources plus populaires, comme le cinéma hollywoodien ou celui de Hitchcock, ainsi qu'une chanson populaire qui raconte l'histoire de la femme d'un gangster qui s'est suicidée dans une chambre portant le numéro 202.

8 Alyce Mahon a conclu son intervention sur l'idée qu'avec son surréalisme idiosyncratique, à la fois américain et transatlantique, et féminin et émancipé sans être féministe, Tanning complique l'histoire du surréalisme comme mouvement qui privilégie les artistes masculins, blancs et européens, et transforme la femme en objet. Elle a également mis l'accent sur l'idée que Tanning résiste à la linéarité, à la fois dans son parcours biographique et dans son œuvre où le labyrinthe occupe une place de choix. 


\section{BIBLIOGRAPHIE}

MAHON, Alyce, dir. Dorothea Tanning : Behind the Door, Another Invisible Door. Madrid : Museo Nacional Centro de Arte Reina Sofía, 2018.

TANNING, Dorothea. Birthday. Santa Monica : Lapis Press, 1986.

\section{NOTES}

1. L'Institut vient de lancer le "Giacometti Lab», un nouvel espace avoisinant qui accueille les programmes pédagogiques de son École des modernités.

2. Parmi les manifestations récentes qui touchent au domaine anglophone, on peut citer l'exposition Flora (5 avril-9 juin 2019), consacrée à une installation très originale créée par un duo d'artistes contemporains américano-suisse, Teresa Hubbard et Alexander Birchler, qui essaient de reconstruire, à travers deux films, le parcours d'une artiste américaine oubliée de l'histoire, Flora Mayo, qui fut l'amante et le modèle de Giacometti. La journée d'études «Le destin des femmes artistes à la période moderne : artistes femmes passées par les académies d'art à Paris au début du XXe siècle » (22 mai 2019) explorait la présence de plusieurs artistes anglo-américaines à Paris, dont par exemple Mina Loy et Berenice Abbott.

3. Mahon a expliqué que Tanning refusait parfois que son art soit considéré comme « surréaliste ».

4. Par exemple, Mahon a précisé que tout au long de sa vie, Tanning refusait de participer à des expositions qui réunissaient uniquement les œuvres d'artistes femmes, à l'exception de sa toute première contribution à une exposition collective, The Exhibition by 31 Women, organisée par Peggy Guggenheim dans sa galerie Art of this Century à New York en 1943, qui était censée n'inclure que 30 femmes, mais fait place à une $31^{\mathrm{e}}$ femme après la découverte de l'art de Tanning. 5. Pour des reproductions des œuvres mentionnées ici, voir le site: https:// www.dorotheatanning.org.

6. Cette traversée transatlantique sera la première de toute une série de migrations, car, comme l'a souligné Mahon, Tanning avait une approche «migrante» de la vie et changeait souvent de résidence avec son mari Max Ernst.

7. Tanning était aussi écrivaine.

8. Le catalogue de l'exposition dont Alyce Mahon est commissaire s'intitule Dorothea Tanning: behind the door, another invisible door.

9. Alyce Mahon a précisé que c'est à Max Ernst qu'on doit le titre de ce tableau. C'est aussi l'occasion de sa première rencontre avec Ernst, qui va voir le tableau sur les conseils de Julien Levy.

\section{INDEX}

Thèmes : Actualité de la recherche 
AUTEUR

YASNA BOZHKOVA

Université Sorbonne Nouvelle - Paris 3 